\title{
An immune response gene expression module identifies a good prognosis subtype in estrogen receptor negative breast cancer Andrew E Teschendorff*, Ahmad Miremadi ${ }^{\dagger}$, Sarah E Pinder ${ }^{\dagger}$, Ian O Ellis ${ }^{\ddagger}$ and Carlos Caldas ${ }^{* \dagger}$
}

Addresses: *Breast Cancer Functional Genomics Laboratory, Cancer Research UK Cambridge Research Institute and Department of Oncology, University of Cambridge, Robinson Way, Cambridge CB2 oRE, UK. ${ }^{+}$Cambridge Breast Unit, Addenbrookes Hospital, Cambridge University Hospitals NHS Foundation Trust, Hills Road, Cambridge, CB2 OQQ, UK. *Histopathology, Nottingham City Hospital NHS Trust and Department of Pathology, University of Nottingham, Nottingham NG5 1PB, UK.

Correspondence: Andrew E Teschendorff. Email: aet21@cam.ac.uk. Carlos Caldas. Email: cc234@cam.ac.uk

\section{Published: 2 August 2007}

Genome Biology 2007, 8:RI57 (doi:10.1 186/gb-2007-8-8-r/57)

The electronic version of this article is the complete one and can be found online at http://genomebiology.com/2007/8/8/RI57
Received: 14 March 2007

Revised: 25 June 2007

Accepted: 2 August 2007

\section{(c) 2007 Teschendorff et al.; licensee BioMed Central Ltd.}

This is an open access article distributed under the terms of the Creative Commons Attribution License (http://creativecommons.org/licenses/by/2.0), which permits unrestricted use, distribution, and reproduction in any medium, provided the original work is properly cited.

\begin{abstract}
Background: Estrogen receptor (ER)-negative breast cancer specimens are predominantly of high grade, have frequent $\mathrm{p} 53$ mutations, and are broadly divided into HER2-positive and basal subtypes. Although ER-negative disease has overall worse prognosis than does ER-positive breast cancer, not all ER-negative breast cancer patients have poor clinical outcome. Reliable identification of ERnegative tumors that have a good prognosis is not yet possible.

Results: We apply a recently proposed feature selection method in an integrative analysis of three major microarray expression datasets to identify molecular subclasses and prognostic markers in ER-negative breast cancer. We find a subclass of basal tumors, characterized by over-expression of immune response genes, which has a better prognosis than the rest of ER-negative breast cancers. Moreover, we show that, in contrast to ER-positive tumours, the majority of prognostic markers in ER-negative breast cancer are over-expressed in the good prognosis group and are associated with activation of complement and immune response pathways. Specifically, we identify an immune response related seven-gene module and show that downregulation of this module confers greater risk for distant metastasis (hazard ratio 2.02, 95\% confidence interval 1.2-3.4; $P=0.009$ ), independent of lymph node status and lymphocytic infiltration. Furthermore, we validate the immune response module using two additional independent datasets.
\end{abstract}

Conclusion: We show that ER-negative basal breast cancer is a heterogeneous disease with at least four main subtypes. Furthermore, we show that the heterogeneity in clinical outcome of ERnegative breast cancer is related to the variability in expression levels of complement and immune response pathway genes, independent of lymphocytic infiltration. 


\section{Background}

It is widely recognized that estrogen receptor (ER)-positive $\left(\mathrm{ER}^{+}\right)$and ER-negative (ER-) breast cancers are two different disease entities. Generally, ER- tumours tend to be of high grade, are more frequently p53 mutated, and have worse prognosis compared with $\mathrm{ER}^{+}$disease. Moreover, while $\mathrm{ER}^{+}$ disease can be treated with hormone therapy, the only targeted therapy available for ER- patients is a monoclonal antibody that binds to the $E R B B 2$ receptor and that is effective only for those ER- tumours with HER2/ERBB2 over-expression.

In spite of these clinical advances, $\mathrm{ER}^{+}$and ER-breast cancers remain heterogeneous diseases, and little is known regarding why patients with the same histopathologic characteristics may have widely different clinical outcomes [1]. This is particularly true for the basal subtype of ER-breast cancer, which is commonly defined by over-expression of cytokeratin markers (CK 5/6 and CK 14) and which is often also HER2 negative (HER2-) [2]. Most recent efforts to obtain a molecular understanding of the observed heterogeneity have focused on ER ${ }^{+}$ breast cancer, where gene expression signatures that are either prognostic or predictive of response to hormone therapy have been derived [3-10]. In contrast, few studies have thus far attempted to derive a prognostic signature within ERbreast cancer. Although cytokeratin markers have been shown to correlate with poor prognosis in breast cancer [1114], an attempt to correlate basal markers with survival within ER- disease has shown that these markers were not predictive of outcome [15]. Based on our work presented here, it appears that the prognostic 'signal' in ER-breast cancer is much weaker than that in $\mathrm{ER}^{+}$disease. This precludes the use of traditional supervised approaches, which assume a sufficiently low false discovery rate (FDR) for deriving gene expression based classifiers. A similar observation was reported by others [16].

Recently, we proposed a novel feature selection method (Profile Analysis using Clustering and Kurtosis [PACK]) [17], that selects genes using a pattern recognition method and that may significantly reduce the FDR. Using PACK in an integrated cohort of $186 \mathrm{ER}^{-}$samples and 1,200 genes, we were able to identify distinct molecular subtypes, including a good prognosis subclass characterized by over-expression of immune response genes. However, these results were not validated in external cohorts.

The purpose of this work is to extend our preliminary findings [17] by applying PACK to the same three breast cancer datasets $[5,9,18]$, but now using a much larger set of common genes (we rescued a significantly larger number of genes from our study [9] by imputing missing data, which led to a much larger number of overlapping genes, approximately 5,000, with the other two arrays.), and to further validate our findings using two external independent cohorts [7,19]. More generally, our goal is to elucidate the molecular taxonomy of
ER-breast cancer and, if possible, to find different prognostic subclasses and the corresponding prognostic markers.

\section{Results}

\section{The FDR is higher in ER- breast cancer}

To understand why in ER-breast cancer it has not been possible to derive a validated prognostic signature using conventional approaches, we compared the FDR for $\mathrm{ER}^{+}$and $\mathrm{ER}^{-}$ disease. We used as cohorts integrated datasets obtained by merging together three of the largest profiled breast cancer cohorts $[5,9,18]$ using the $z$-score transformation, a procedure that we validated previously [17]. Briefly, the z-score transformation shifts the mean of each gene expression vector in each cohort to zero, while scaling its variance to unity. The transformed gene expression vectors are then merged across cohorts. This merging step resulted in integrated expression matrices of $186 \mathrm{ER}^{-}$and $527 \mathrm{ER}^{+}$tumors profiled over a common set of 5,007 genes. To enable the comparison, we selected at random $186 \mathrm{ER}^{+}$tumors from the 527 available. We then used the univariate Cox proportional hazards model with time to distant metastasis (TTDM) and overall survival as end-points to obtain $P$ values of significance for all the genes. Next, we estimated, for each choice of significance threshold, the number of false positives using the q-value approximation [20]. The numbers of significant genes and false positives as a function of the significance threshold were then plotted for $\mathrm{ER}^{+}$and ER-breast cancer and for the two different end-points used (Figure 1). We verified that the curves for $\mathrm{ER}^{+}$breast cancer were robust to random selections of the $186 \mathrm{ER}^{+}$tumors. This showed that the FDR is much higher in ER-tumours and motivated us to develop a different feature selection approach based on a pattern recognition algorithm (PACK) [17].

\section{Finding ER- subclasses using PACK}

If the aim is to identify subclasses within a tumor type, then it is natural that unsupervised methods be applied to sample sets that are composed entirely of this tumor type. In fact, given the hierarchical $\mathrm{ER}^{+} / \mathrm{ER}^{-}$- subdivision for breast cancer, it is questionable whether $\mathrm{ER}^{-}$subgroups can be correctly defined based on clusters that were derived by using both $\mathrm{ER}^{+}$ and ER- samples, as was done in other studies (for example [21-23]). To see this, assume that all tumor types and genes are used in the clustering algorithm. It is very likely that the clusters within ER- tumors reflect not only the interesting variability of genes within ER- tumors but also the variability of genes that are important for classification of $\mathrm{ER}^{+}$tumors. The variability of these genes in ER-tumors may represent undesired noise, which, if not removed, can affect the inferred clusters. Thus, in order to identify relevant subtypes of ERtumors more robustly, we decided to use ER- tumors only. Moreover, in view of the relatively high FDR in ER- disease, we decided to apply the PACK methodology, which has already been shown to provide a more reliable identification of molecular classifiers [17]. Because PACK requires large 


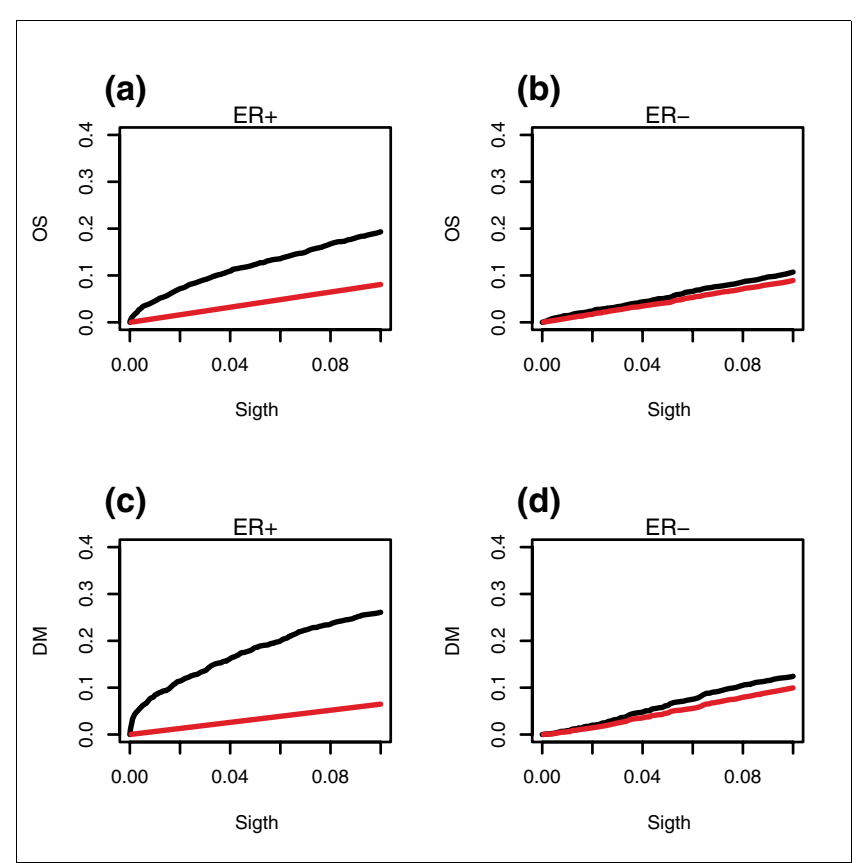

Figure I

FDR comparison in ER- and $\mathrm{ER}^{+}$breast cancer. For various significance thresholds (sigth), we plot the fraction of observed genes with $P$ values less than the significance threshold (black) as well as the corresponding fraction of false positives, as estimated using a q value analysis (red). (a) Overall survival for $\mathrm{ER}^{+}$breast cancer. (b) Overall survival for ER-breast cancer. (c) Time to distant metastasis for $E R^{+}$breast cancer. (d) Time to distant metastasis for ER-breast cancer. $P$ values were obtained from the log-rank test using Cox regression models. ER, estrogen receptor; FDR, false discovery rate.

sample sizes (for small sample sizes, kurtosis estimates can have large variance and clustering algorithms that aim to predict the optimal number of clusters have a high false negative rate), we applied it to the integrated data matrix derived previously. Two additional independent ER- cohorts $[7,19]$ were left out to serve as validation studies. The five microarray datasets used are summarized in Table 1 by platform type, number of ER-samples, and number of poor outcome events.

Briefly, we review the concepts that underpin PACK (see Materials and methods, below, and Figure 2). The hypothesis is that genes that play an important role as classifiers or biomarkers are more likely to have expression profiles that are mixtures of gaussian distributions. On the other hand, false positives, in spite of their spurious association with a phenotype, are less likely to be described by a mixture of distributions. Thus, selecting genes based on whether they have structure in their expression profiles is likely to pick out the relevant markers from those that are just false positives. Next, we propose to focus on those genes that define the largest subgroups. Although genes that define small subgroups are also of interest, it is natural to identify first those genes that define the largest subclasses. While such features can be found from the inferred cluster sizes, it turns out that such features are generally also characterized by a negative (or close to zero) kurtosis profile (see Materials and methods, below) [17]. As shown previously, negative kurtosis expression profiles are in effect a mixture of at least two (gaussian) distributions of approximately equal weights. Thus, by selecting those genes that have the most negative kurtosis expression profiles, we have identified the markers that define the largest subclasses within the sample set (Figure 2). It is clear that many of these features will be highly correlated because they define almost the same subclasses. It follows that further application of traditional clustering algorithms over these negative kurtosis profiles will enable reliable identification of the major subclasses within the sample set. We note that because we are interested in the most negative kurtosis profiles and because the clusters in the individual gene profiles are only needed to study the cluster distribution of phenotypes, the cluster inference step on the individual gene profiles (known as PAC) can be performed after computation of kurtosis (known as PAK) on the selected subset of negative kurtosis profiles (Figure 2).

Table I

\begin{tabular}{|c|c|c|c|c|c|c|}
\hline \multirow[t]{2}{*}{ Ref. } & \multirow[t]{2}{*}{ Cohort name } & \multirow[t]{2}{*}{ Oligo microarray platform } & \multicolumn{2}{|r|}{ ER- } & \multicolumn{2}{|r|}{$\mathrm{ER}^{+}$} \\
\hline & & & $n$ & $\begin{array}{l}\text { Death/distant } \\
\text { metastasis }(n)\end{array}$ & $n$ & $\begin{array}{l}\text { Death/distant } \\
\text { metastasis }(n)\end{array}$ \\
\hline$[18]$ & NKI2 & Agilent & 69 & 34 & 226 & 45 \\
\hline$[5]$ & EMC & Affymetrix & 77 & 27 & 208 & 80 \\
\hline [9] & $\mathrm{NCH}$ & Agilent & 40 & 14 & 93 & 21 \\
\hline [19] & UPP & Affymetrix & 34 & 6 & 213 & 49 \\
\hline [7] & $\mathrm{JRH}-2$ & Affymetrix & 24 & 6 & 72 & 17 \\
\hline
\end{tabular}

$E R$, estrogen receptor. 
(a)

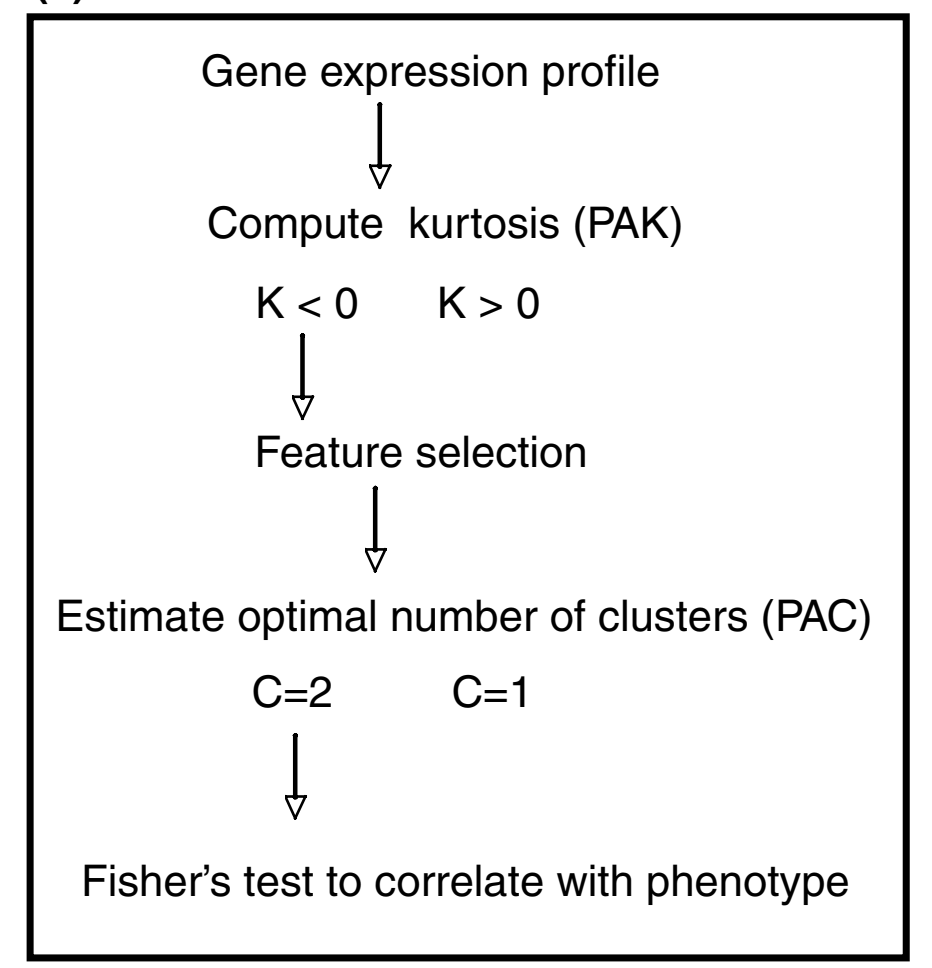

(b) Bi-modal: major subgroups
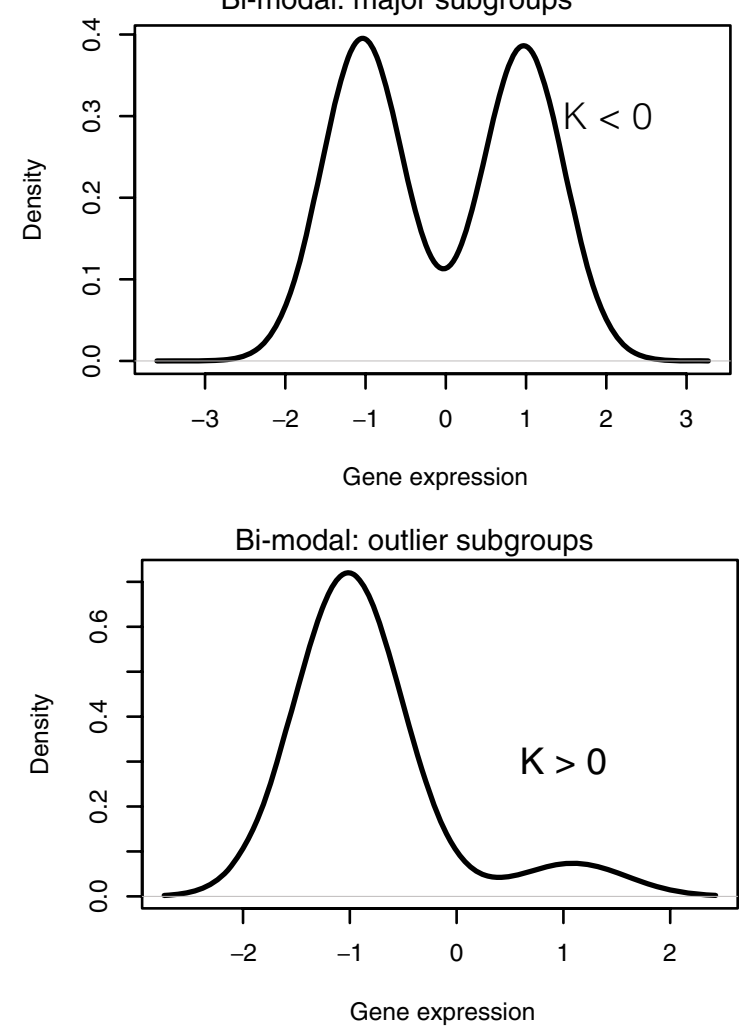

Figure 2

PACK flowchart. (a) A schematic diagram of PACK, as used in this study. For each gene expression profile an unbiased estimate of its kurtosis, K, is computed. Genes with negative kurtosis are selected because only these define large subgroups (of sizes $>22 \%$ of the total sample size). Further unsupervised clustering may then be performed on this subset of negative kurtosis profiles to find novel tumor subclasses. Alternatively, to find robust prognostic markers, negative kurtosis profiles are filtered further based on whether there is evidence of bimodality $(C=2)$. This step requires a cluster inference algorithm and a model selection criterion to discard those profiles that are best described by a single gaussian $(C=I$; by random chance gaussian profiles may have negative kurtosis). Correlation to phenotypes (here phenotypes) is done with Fisher's test to evaluate whether the distribution of the categorical phenotype across the two clusters is significantly different from random. (b) Density curves of typical bimodal negative and positive kurtosis gene expression profiles. X-axis shows gene expression on a log2 scale. PACK, Profile Analysis using Clustering and Kurtosis.

\section{Distinct molecular subgroups of ER-breast cancer}

Applying PAK to the integrated ER- data matrix of 5,007 genes, we found 813 genes with a negative kurtosis profile (Additional data file 1). Interestingly, applying the same analysis to $\mathrm{ER}^{+}$breast cancer, we found only a much smaller number (193) of negative kurtosis profiles (Additional data file 2), despite there being roughly twice as many bimodal profiles in $\mathrm{ER}^{+}$breast cancer (about 4500 in $\mathrm{ER}^{+}$versus about 2,500 in $\mathrm{ER}^{-}$). We verified by explicit simulation that the significantly lower proportion of negative kurtosis profiles in $\mathrm{ER}^{+}$disease could not be explained by the larger sample size in $\mathrm{ER}^{+}$(527) compared with $\mathrm{ER}^{-}$disease (186; data not shown).

Having identified the relevant features, we next clustered the ER- tumors over these. Using hierarchical clustering with Pearson correlation metric and complete linkage, we found that samples clustered into five main groups, each character- ized by the expression patterns of four gene clusters that were found to be strongly enriched for specific gene ontologies (Figure 3a and Additional data file 3). One group was characterized by over-expression of $E R B B 2$, the steroid hormone receptor $A R$, and genes related to steroid estrogen response (such as GATA3, TFF1, and DNALI1). This subtype is therefore similar to the apocrine subclass, recently proposed $[24,25]$, which is characterized by over-expression of $A R$ and genes that are either direct targets of ER or responsive to estrogen [24,25]. Thus, we decided to call this ER- subtype (over-expressing steroid response genes) ' $\mathrm{SR}^{+}$', although it is clear that it also defines the well known HER2 ${ }^{+}$subtype.

The other four groups were characterized mainly by absent or lower expression of these steroid response genes. One of these four clusters was characterized by over-expression of genes related to cell cycle and cell proliferation pathways $\left(\mathrm{CC}^{+}\right)$, and another cluster also had over-expression of immune response 
genes $\left(\mathrm{CC}^{+} / \mathrm{IR}^{+}\right)$. For the remaining two clusters, one was characterized by over-expression of extracellular matrix genes $\left(\mathrm{ECM}^{+}\right)$, and the other was characterized by overexpression of immune response genes $\left(\mathrm{IR}^{+}\right)$only.

\section{Relation to the intrinsic subtype classification}

In order to relate the five identified molecular subtypes to the intrinsic breast cancer classification [21,26], we used the recently validated single sample predictor (SSP) [23] to classify the $186 \mathrm{ER}^{-}$samples into the various intrinsic subtypes (Figure $3 \mathrm{a}$ and Table 2). In addition, we studied the expression profiles of recently validated basal markers [27] and genes in the $E R B B 2$ amplicon across the five identified clusters (Figure 3a and Additional data file 4). Based on these figures and Table 2, we could draw the following conclusions. First, the $\mathrm{SR}^{+}$cluster was highly correlated with the usual $\mathrm{HER}^{+}$intrinsic subtype. Second, the $\mathrm{CC}^{+} / \mathrm{IR}^{+}$and $\mathrm{CC}^{+}$clusters defined distinct subtypes of basal tumors. Third, the $\mathrm{ECM}^{+}$cluster was mostly basal, but it contained a relatively high proportion of normal-like and luminal-A-like ERtumors; it also exhibited the most varied grade distribution, with most low-grade ER- tumors falling into this class. Finally, the main constituents of the $\mathrm{IR}^{+}$cluster were basal and HER2 ${ }^{+}$.

It further follows from these observations that ER- normal and luminal A samples were predominantly characterized by over-expression of ECM genes. ER-basal tumors, on the other hand, exhibited a more complex pattern and appeared to divide into at least four subgroups $\left(\mathrm{CC}^{+} / \mathrm{IR}^{+}, \mathrm{CC}^{+}, \mathrm{ECM}^{+}\right.$, and $\mathrm{IR}^{+}$.

To investigate further the relation of our five ER- subclasses with the intrinsic subtypes, we considered to which ER- subclass $\mathrm{ER}^{+}$samples of known intrinsic subtype were most similar. To this end we first constructed, for each of the five ERsubclasses, mean centroids over the 813 negative kurtosis genes (Additional data file 5). To validate the centroids, the same ER- samples were assigned a subclass using a nearest centroid criterion (samples for which the largest Pearson correlation coefficient was <0.25 were considered unclassified; see Materials and methods, below), which showed that 156 (84\%) were classified, of which 143 (92\%) were assigned the correct subclass. Next, using the SSP to classify the $527 \mathrm{ER}^{+}$ samples into the intrinsic subtypes, we then assigned each of the $527 \mathrm{ER}^{+}$samples into an ER- subclass based on the same nearest centroid criterion (Table 3 ). As expected, only $4 \%$ of $\mathrm{ER}^{+}$tumors were classified as basal, whereas the majority (82\%) of them were luminal. Moreover, the analysis showed that $\mathrm{ER}^{+}$luminal $\mathrm{B}$ samples were most similar to $\mathrm{CC}^{+}$and $\mathrm{CC}^{+} / \mathrm{IR}^{+} \mathrm{ER}-$ samples, which is consistent with the fact that all of these samples over-express cell cycle and cell proliferation genes. In contrast, $\mathrm{ER}^{+}$luminal A samples were most similar to $\mathrm{ECM}^{+}(63 \%), \mathrm{IR}^{+}$(26\%), and $\mathrm{SR}^{+}$(8\%) ER- samples. Not surprisingly, almost all (16/19 [84\%]) 'normal' ER+ samples were most similar to $\mathrm{ECM}^{+} \mathrm{ER}^{-}$samples. All basal ER+ sam- ples had expression profiles most similar to $\mathrm{CC}^{+} / \mathrm{IR}^{+}$and $\mathrm{CC}^{+}$ subtypes. Interestingly, only 16 of the 42 (38\%) ER ${ }^{+} \mathrm{HER}^{+}$ tumors exhibited significantly correlated expression profiles to any one of the five ER- subclasses, with most of these (11) mapping to the $\mathrm{CC}^{+} / \mathrm{IR}^{+}$subtype.

\section{A subgroup of good prognosis in ER-breast cancer}

We next considered whether the five identified clusters were associated with different prognostic groups. Because we had merged different cohorts, and it is questionable whether survival data can be also merged together, we decided to test first the clusters for association with clinical outcome by using a dichotomized outcome variable. Specifically, poor outcome was defined as any death or distant metastasis event, whereas good outcome was defined as a patient alive or with no distant metastasis. By studying the distribution of good and poor outcome events in the respective clusters, significant associations with prognosis could be found by means of Fisher's exact test (Table 2). Interestingly, this showed that the $\mathrm{IR}^{+}$ subgroup had better prognosis when compared with the $\mathrm{ECM}^{+}(P=0.08), \mathrm{CC}^{+}(P=0.03)$, and $\mathrm{SR}^{+}(P=0.005)$ subclasses.

Compared with the $\mathrm{CC}^{+} / \mathrm{IR}^{+}$subgroup it also had better prognosis, although the difference was not statistically significant $(P=0.19)$. We thus combined the $\mathrm{CC}^{+} / \mathrm{IR}^{+}$and $\mathrm{IR}^{+}$subclasses together and evaluated the prognosis of this larger subclass relative to the rest of $\mathrm{ER}^{-}$samples. Consistent with our previous result [17] we found that the ER- tumors overexpressing immune response genes had better prognosis than ER-samples that did not $(P=0.02)$.

To further confirm our findings, we also generated KaplanMeier survival curves for the five identified subclasses using TTDM as the end-point (Figure $3 \mathrm{~b}$ ). This showed that the $\mathrm{SR}^{+}$ and $\mathrm{CC}^{+}$subclasses had worst prognosis, whereas the $\mathrm{IR}^{+}$subclass was the group with best prognosis. Specifically, relative to the $\mathrm{IR}^{+}$subclass the $\mathrm{SR}^{+}$subgroup had a hazard ratio (HR) of 3.70 (95\% confidence interval [CI] 1.49 to 9.24; $P=0.005$ ), whereas the $\mathrm{CC}^{+}$subgroup had an HR of 2.75 (95\% CI 1.07 to 7.05; $P=0.035)$. Similarly, relative to the $\mathrm{CC}^{+} / \mathrm{IR}^{+}$subclass, the $\mathrm{SR}^{+}$subgroup had an $\mathrm{HR}$ of 2.35 (95\% CI 1.13 to 4.88 ; $P=0.02$ ), whereas for the $\mathrm{CC}^{+}$subgroup it did not quite reach statistical significance (HR 1.80, 95\% CI 0.83 to 3.89 ; $P=0.13)$. We verified the statistical significance of these survival differences by 10,000 random permutations of the samples, which showed that the theoretical $P$ value estimates above were essentially identical to the empirically derived $P$ values.

\section{Prognostic markers in ER- tumors are associated with immune response functions}

The better prognosis of the $\mathrm{IR}^{+}$subclass is likely due to specific genes that are individually prognostic. In order to find these, we applied the PAC algorithm to the 813 genes with negative kurtosis expression profiles. Briefly, this procedure 


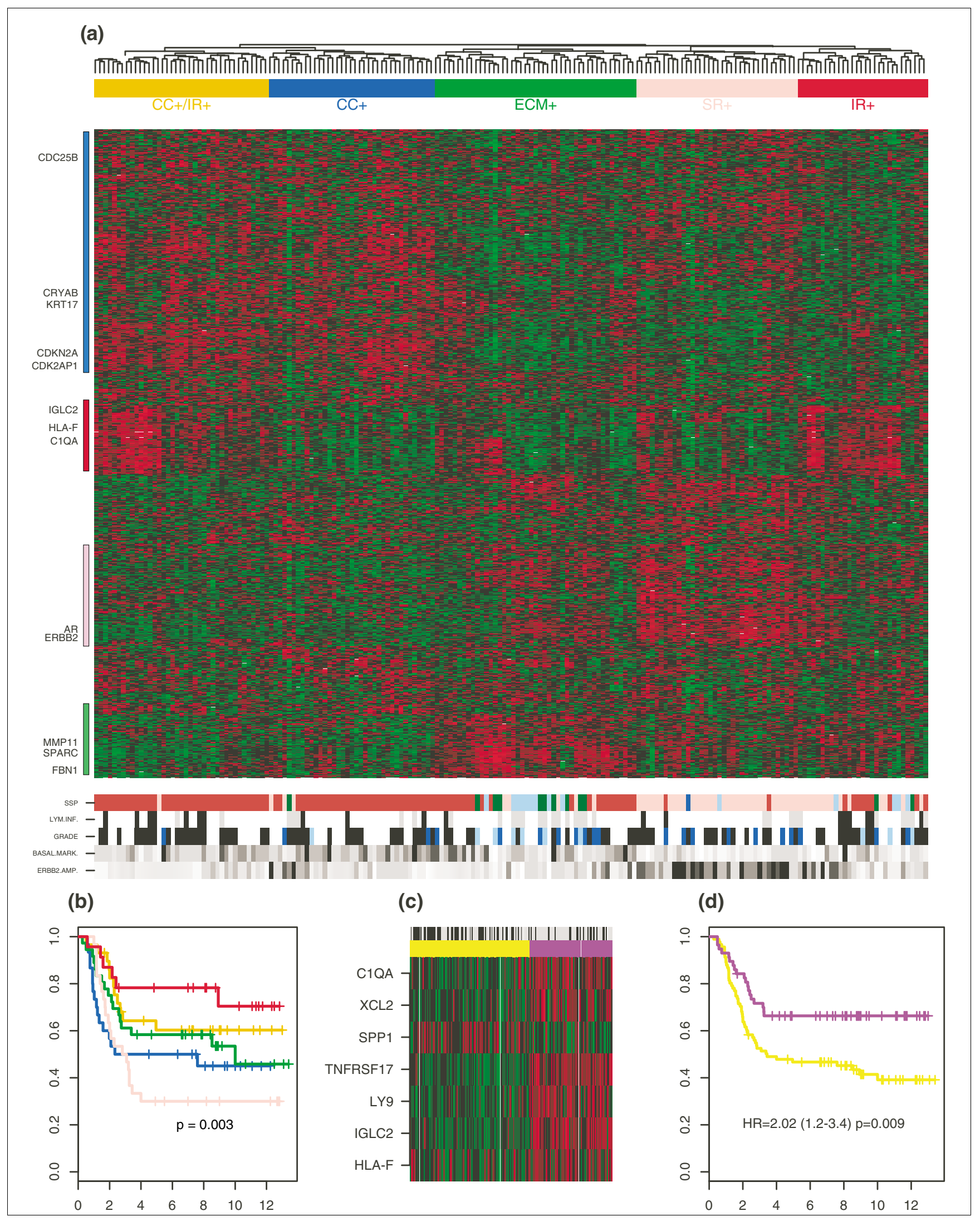

Figure 3 (see legend on next page) 
Figure 3 (see previous page)

Molecular subclasses in ER- breast cancer. (a) Complete linkage hierarchical clustering of I86 ER-breast tumors over 813 genes with negative kurtosis profiles. Five sample clusters were identified and characterized in terms of the patterns of over-expression and under-expression of four gene clusters related to cell cycle (CC; blue), immune response (IR; red), extracellular matrix (ECM; green), and steroid hormone response (SR; pink) functions. Panels show the distribution of the SSP subtype [23], the lymphocytic infiltration score, histologic grade, basal marker [27], and ERBB2 ${ }^{+}$amplifier subtype. Panel color codes: SSP (pink = HER2, brown = basal, dark green = normal, sky blue = luminal A, and blue = luminal B); LYM.INF (black = high, gray = low, and white = missing); GRADE (black = high, blue $=$ intermediate, sky blue $=$ low, and white $=$ missing), BASAL.MARK. $($ black $=$ high and white $=$ low), ERBB2AMP $($ black $=$ high and white $=$ low). The BASAL.MARK. profile represents an average over validated basal markers in [27], whereas the ERBB2-AMP profile was calculated as an average over three genes in the ERBB2 amplicon (ERBB2, STARD3, GRB7). (b) Kaplan-Meier curves for time to distant metastasis (years) and for the five subclasses identified in panel (a). (c) Partitioning around medoids clustering over the seven-gene prognostic immune response module. Panel color codes: purple = cluster over-expressing module, yellow = cluster under-expressing module, black = poor outcome samples, gray $=$ good outcome samples, green = relative under-expression, and red = relative over-expression. (d) Kaplan-Meier curves for time to distant metastasis for the two groups identified in panel (c). Hazard ratio, 95\% confidence interval, and log-rank test $P$ values are shown. ER, estrogen receptor; SSP, single sample predictor.

selects features based on whether their expression profiles are best described as a mixture of gaussians or not, and then evaluates whether the distribution of good and poor outcome events among the inferred clusters is statistically different from random or not. Applying PAC, we found 22 genes with a Fisher test $P<0.05$ (Additional data file 1). A Gene Ontology (GO) enrichment analysis of these 22 genes using the GOTree
Machine [28] showed that immune/defense response was the most enriched GO, with seven genes falling into this category (C1QA, IGLC2, LY9, TNFRSF17, SPP1, XCL2, and HLA-F). The expression profiles for these seven genes confirmed the presence of distinct clusters with nonrandom distributions of good and poor outcome samples (Figure 4 provides detailed profiles of $C 1 Q A$ and $I G L C 2)$.

Table 2

Distribution of ER- samples among clusters, prognostic groups and intrinsic subtypes

\begin{tabular}{|c|c|c|c|c|c|c|c|c|c|c|}
\hline \multirow[t]{2}{*}{ Cluster } & \multirow[t]{2}{*}{$n$} & \multicolumn{2}{|c|}{ Outcome } & \multicolumn{2}{|c|}{$\mathrm{LI}$} & \multicolumn{5}{|c|}{ ER- } \\
\hline & & Good & Poor & High & Low & Luminal B & Luminal $\mathrm{A}$ & Normal & Basal & HER2 \\
\hline $\mathrm{CC}^{+} / \mathrm{IR}^{+}$ & 39 & 25 & 14 & 7 & 2 & 0 & 0 & 0 & 38 & 1 \\
\hline $\mathrm{CC}^{+}$ & 37 & 18 & 17 & 3 & 5 & 0 & 0 & 1 & 33 & 3 \\
\hline $\mathrm{ECM}^{+}$ & 45 & 26 & 18 & 1 & 14 & 0 & 9 & 9 & 22 & 5 \\
\hline $\mathrm{SR}^{+}$ & 36 & 16 & 20 & 2 & 4 & 1 & 1 & 0 & 2 & 32 \\
\hline $\mathrm{IR}^{+}$ & 29 & 23 & 6 & 6 & 6 & 0 & 4 & 2 & 9 & 14 \\
\hline
\end{tabular}

For the five clusters of Figure 2a, we give the number of samples per cluster $(n)$ and their distributions over good/poor outcome, high/low lymphocytic infiltration (LI) score, and intrinsic subtypes as determined by the single sample predictor (SSP) classifier. Clusters were labeled by the main Gene Ontology of genes over-expressed in the group: $\mathrm{CC}^{+}$(cell cycle), $\mathrm{CC}^{+} / \mathrm{IR}^{+}$(cell cycle and immune response), $\mathrm{ECM}^{+}$(extracellular matrix), $\mathrm{SR}^{+}$(steroid hormone response), and $\mathrm{IR}^{+}$(immune response). ER, estrogen receptor.

Table 3

Classification of ER ${ }^{+}$intrinsic subtypes, medullary breast cancer, and BRCAI tumors into ER- subclasses

\begin{tabular}{|c|c|c|c|c|c|c|c|c|}
\hline & \multicolumn{5}{|c|}{$\mathrm{ER}^{+}$} & \multirow[t]{2}{*}{$M B C$} & \multirow[t]{2}{*}{ DBC } & \multirow[t]{2}{*}{ BRCAI } \\
\hline & Luminal B & Luminal A & Normal & Basal & HER2 & & & \\
\hline$n$ & 97 & 337 & 32 & 19 & 42 & 22 & 44 & 18 \\
\hline$n$ classifiable & 37 & 113 & 19 & 17 & 16 & 20 & 33 & 16 \\
\hline $\mathrm{CC}^{+} / \mathrm{IR}^{+}$ & 17 & 2 & 0 & 13 & 11 & 14 & 4 & 13 \\
\hline $\mathrm{CC}^{+}$ & 20 & 2 & 0 & 4 & 1 & 3 & 3 & 2 \\
\hline $\mathrm{ECM}^{+}$ & 0 & 71 & 16 & 0 & 0 & 1 & 3 & 1 \\
\hline $\mathrm{SR}^{+}$ & 0 & 9 & 0 & 0 & 0 & 0 & 20 & 0 \\
\hline $\mathrm{IR}^{+}$ & 0 & 29 & 3 & 0 & 4 & 2 & 3 & 0 \\
\hline
\end{tabular}

Classification of $\mathrm{ER}^{+}$intrinsic subtypes ( 527 samples from the integrated cohort NKI2 + EMC + NCH), 22 medullary breast cancers (MBCs) and 44 ductal breast cancers (DBCs) from [38], and I8 BRCAI mutants from [3] into ER- subclasses, using the nearest centroid criterion with Pearson correlation as distance metric. Only samples with Pearson correlation coefficients larger than 0.25 were deemed classifiable. ER, estrogen receptor. 


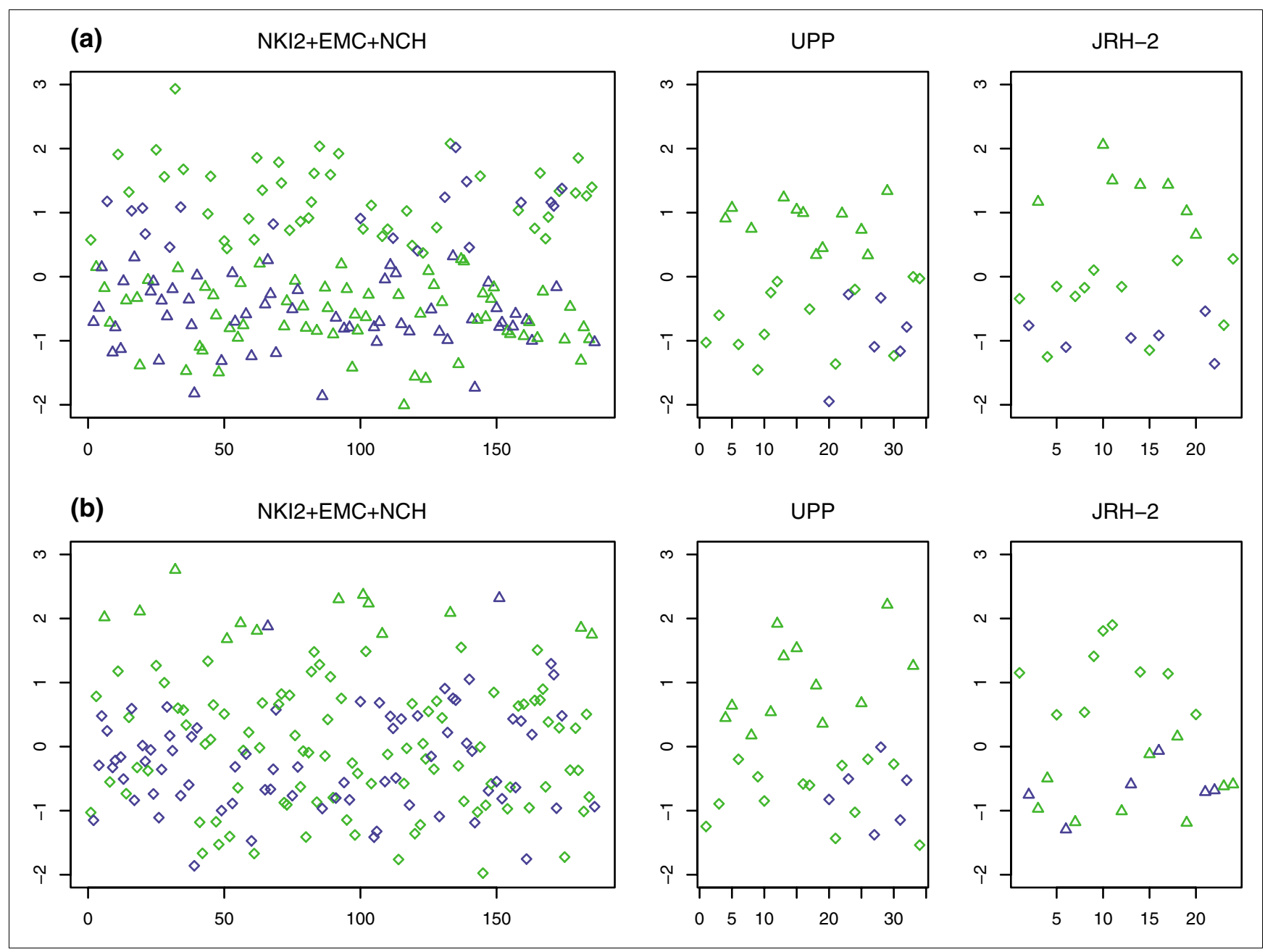

Figure 4

Expression profiles of selected prognostic markers in ER-breast cancer. Expression profile (on a log2 scale) of selected prognostic markers (a) IGLC2 and (b) CIQA in the integrated cohort of 186 ER- tumours (NKI2 + EMC + NCH), and in the validation cohorts UPP and JRH-2. Good outcome samples are shown in green, and poor outcome samples in blue. Clusters were inferred using the variational Bayesian approach in NKI2 + EMC $+\mathrm{NCH}$ and the pam algorithm in the UPP and JRH-2 cohorts. Infered clusters are indicated by different shapes (triangles and diamonds). ER, estrogen receptor; pam, partitioning around medoids.

In contrast, application of the same method to $\mathrm{ER}^{+}$breast cancer yielded 29 genes (out of a possible 193) with $P<0.05$ (Additional data file 2). In spite of there being only 29 genes in this list, there were many with mitotic cell cycle functions, notably $U B E 2 C, M A D 2 L 1, E 2 F 1$ and $K I F C 1$, and GO enrichment analysis confirmed that the cell cycle GO was the most significantly enriched category followed by transcription regulatory activity. This result for $\mathrm{ER}^{+}$breast cancer confirms findings reported elsewhere that poor prognosis in $\mathrm{ER}+$ breast cancer is related mainly to over-expression of genes in cell cycle and cell proliferation pathways [3,5,7]. Notably, none of the identified prognostic genes were related to immune response functions.

Having identified a prognostic module of seven immune response genes (henceforth called the IR module), we next confirmed that clustering over this module resulted in clus- ters significantly associated with clinical outcome. Specifically, the partitioning around medoids (pam) clustering algorithm [29] with two centers predicted one cluster with 52 good outcome and 56 poor outcome patients, and another cluster with 56 good outcome and only 19 poor outcome events, which was highly significant under Fisher's exact test $(P=0.0004$; Figure $3 c)$. Kaplan-Meier and Cox regression analyses further confirmed that the group not over-expressing the seven-gene module (out of the seven genes the majority [six] are not over-expressed) had a greater risk for distant metastasis (HR 2.02, 95\% CI 1.2 to 3.4; $P=0.009$; Figure $3 \mathrm{~d}$ ).

\section{Relation to STATI and IFN cluster}

Next, we investigated the relation of the good prognosis subgroup identified here with the novel IFN regulated cluster identified recently [23]. As shown previously [23], the IFN cluster, defined by over-expression of interferon regulated 
genes, including the transcriptional regulator STAT1, was most closely related to the basal subclass and had a prognostic performance similar to that of luminal B samples when compared with the luminal A and normal subtypes. Interestingly, when compared with the basal and HER2 ${ }^{+}$subclasses, the IFN group had better prognosis, although a formal comparison was not conducted by $\mathrm{Hu}$ and coworkers [23].

Among the 98 genes in the immune response gene cluster (Figure $3 \mathrm{a}$ and Additional data file 1), we identified a total of 14 with interferon related functions, including STAT1, SP11O, NFKBI, IFI44, IFNGR1, ISGF $3 G$, and IRF7. Interestingly, however, none of these genes showed association with prognosis except SP11O. Thus, although it appears that our subclass is related to the IFN class discovered by $\mathrm{Hu}$ and coworkers [23], it is also distinct in that the associated prognostic markers are not in the IFN cluster.

\section{Immune response module predicts outcome independently of lymph node status}

Because our IR gene cluster included STAT1 and interferoninduced genes, and these genes have been shown to be associated with lymph node (LN) metastasis [30], we considered whether the subgroup of ER-samples over-expressing the 98gene IR cluster was significantly associated with LN status. Because all patients in the ECM cohort were $\mathrm{LN}$ negative, this analysis was only performed on the NKI2 and NCH cohorts (109 ER- patients, of whom 42 had LN involvement). Using pam clustering with two centers over these 98 genes and 109 samples, we found subgroups with similar proportions of LN metastases (43 LN negative and $28 \mathrm{LN}$ positive versus $24 \mathrm{LN}$ negative and $14 \mathrm{LN}$ positive; Fisher's exact test $P=0.84$ ). Moreover, clustering only over the 14 genes involved in the IFN subcluster still did not show a significantly nonrandom distribution of LN metastases among the clusters $(P=0.23)$, although in agreement with Huang and coworkers [30] the cluster over-expressing the IFN genes had proportionally more LN metastases. Similarly, the distribution of LN metastases among the two clusters predicted by the IR module was not significantly different from random $(P=0.48)$. While LN status itself was a significant predictor of distant metastasis both in univariate (HR 2.28, 95\% CI 1.36 to $3.85 ; P=0.002$ ) and multivariate (HR 2.16, 95\% CI 1.28 to $3.64 ; P=0.004$ ) Cox regression analysis (this result is for a multivariate model including LN status and the IR module as predictors.), importantly, the IR module remained a prognostic predictor for TTDM (HR 1.93, 95\% CI 1.14 to $3.26 ; P=0.015$ ) in the multivariate model that included LN status (Table 4 ). This showed that the IR module identified here adds independent prognostic value over LN status in ER-breast cancer.

\section{Immune response module predicts outcome independently of lymphocytic infiltration}

The upregulation of immune response genes in good prognosis tumors could be explained by the fact that these tumors elicit a stronger immune and inflammatory response, as measured for example by a higher degree of lymphocytic infiltration (LI). The association of high LI with good prognosis is well known [31-34], and although a few conflicting results have also been reported [35-37], we thought it natural to consider whether upregulation of the identified immune response module conferred good prognosis independently of LI. To this end, we scored the samples in the NCH cohort for LI (see Materials and methods, below) and combined these with the available LI score information from the NKI2 cohort, yielding a total of 50 scored samples. We found that although there were proportionally more tumors with high LI scores in the group over-expressing the immune response genes (specifically there were 11 high LI and 9 low LI samples versus 8 high LI and 22 low LI in the under-expressed group; Fisher test, $P=0.07$ ), a multivariate Cox regression with TTDM as the outcome variable and the seven-gene IR module and LI score as predictors showed that the immune response module was still a strong predictor of clinical outcome, independent of LI (HR 2.05, 95\% CI 0.71 to 5.97; $P=0.19$; Table 4). (The $P$ value is only marginally below statistical significance, which is most likely due to the relatively small sample size.) Supporting this result further, we did not find in ER- tumors any significant association between LI and clinical outcome (Table 4).

\section{Relation to medullary and BRCAI ER-breast cancer}

To further confirm the independent prognostic power of the IR module from LI, we investigated the relationship of the identified good prognosis tumors with medullary breast cancers (MBCs), which are characterized by high LI scores and relatively good prognosis [38]. Thus, we considered to which ER- subclass the $22 \mathrm{MBC}$ expression profiles from the report by Bertucci and coworkers [38] were most similar (Table 3). (For completeness and reference, we also performed the analysis for the 44 ductal breast cancers [DBCs], also profiled by Bertucci and coworkers [38].) This showed that of the $22 \mathrm{MBCs}, 20$ showed reasonably strong correlation to one of the ER- subclass centroids, 14 of which (70\%) were most similar to the $\mathrm{CC}^{+} / \mathrm{IR}^{+}$subtype, whereas only two (11\%) were most similar to the $\mathrm{IR}^{+}$subtype. In contrast, for the 33 DBC which could be classified, only four (12\%) and three (9\%) were most similar to the $\mathrm{CC}^{+} / \mathrm{IR}^{+}$and $\mathrm{IR}^{+}$subtypes. These results mirror the distribution of LI scores across the five ERsubclasses (Figure $3 \mathrm{a}$ and Table 2) and further confirms that the best prognostic group $\left(\mathrm{IR}^{+}\right)$is not related to $\mathrm{MBC}$, whereas $\mathrm{CC}^{+} / \mathrm{IR}^{+}$is.

A similar analysis was performed for BRCA1 mutant tumors. Of the 16 BRCA1 mutants from the NKI2 cohort [3], which were also deemed classifiable based on a 0.25 correlation threshold, 13 (81\%) had expression profiles most similar to the $\mathrm{CC}^{+} / \mathrm{IR}^{+}$subtype (Table 3 ). None showed similarity to the $\mathrm{IR}^{+}$subclass. Therefore, this suggests that $\mathrm{ER}^{-} \mathrm{BRCA} 1$ mutants, in common with MBCs, are most similar to the $\mathrm{CC}^{+}$/ $\mathrm{IR}^{+}$subclass identified here. 
Table 4

Univariate and Multivariate Cox-regression model

\begin{tabular}{|c|c|c|c|c|}
\hline & \multicolumn{2}{|c|}{ ER- $(n=186)$} & \multicolumn{2}{|c|}{$\mathrm{ER}^{+}(n=527)$} \\
\hline & $\mathrm{HR}(95 \% \mathrm{Cl})$ & $P$ & $\mathrm{HR}(95 \% \mathrm{Cl})$ & $P$ \\
\hline LN & $2.28(1.36-3.85)$ & 0.002 & $2.07(1.47-2.90)$ & $<10^{-4}$ \\
\hline $\mathrm{LI}$ & $1.06(0.66-1.70)$ & 0.9 & $1.50(0.72-3.14)$ & 0.58 \\
\hline IRM & $2.02(I .19-3.4 I)$ & 0.009 & $1.25(0.9|-| .7 I)$ & 0.19 \\
\hline$L N^{a}+$ IRM & $2.16(1.28-3.64)$ & 0.004 & $2.10(1.49-2.96)$ & $<10^{-4}$ \\
\hline$L N+I R M a$ & $1.93(1.14-3.26)$ & 0.015 & $1.29(0.94-1.76)$ & 0.11 \\
\hline $\mathrm{LI}^{\mathrm{a}}+\mathrm{IRM}$ & $0.86(0.32-2.28)$ & 0.76 & $1.75(0.41-7.47)$ & 0.45 \\
\hline $\mathrm{LI}+\mathrm{IRM}^{\mathrm{a}}$ & $2.05(0.7 \mid-5.97)$ & 0.19 & $0.57(0.27-1.19)$ & 0.13 \\
\hline$L N^{a}+L I+I R M$ & $1.79(0.70-4.62)$ & 0.22 & $1.48(0.68-3.19)$ & 0.32 \\
\hline$L N+L^{a}+I R M$ & $0.84(0.51-1.38)$ & 0.72 & $1.65(0.38-7.07)$ & 0.5 \\
\hline$L N+L I+I R M a$ & $2.22(0.76-6.50)$ & 0.15 & $0.57(0.27-1.20)$ & 0.14 \\
\hline
\end{tabular}

The table summarizes the hazard ratio $(\mathrm{HR}), 95 \%$ confidence interval $(\mathrm{Cl})$, and log-rank test $P$ values of univariate Cox proportional hazards regression models, with lymph node status ( $\mathrm{LN}=\mathrm{I} / 0$ for $\mathrm{LN}+/$-), level of lymphocytic infiltration ( $\mathrm{LI}=\mathrm{I}$ for low infiltration score, and $\mathrm{LI}=0$ for high infiltration score) and the classification based on the seven-gene immune response related module (IRM; $2=$ down-regulation of module, $\mathrm{I}=$

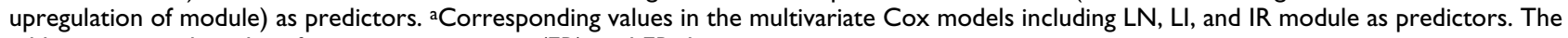
table compares the values for estrogen receptor (ER)- and $\mathrm{ER}^{+}$breast cancer.

\section{External validation}

Having identified a prognostic module related to immune response, we next attempted to validate this finding using two independent breast cancer cohorts $[7,19]$. Specifically, the hypothesis to be tested was that over-expression of the identified prognostic markers is associated with good prognosis, except for $S P P 1$, for which good prognosis is hypothesized to be associated with under-expression. Because of the relatively small sample size of the two external cohorts, an algorithm that attempts to learn the optimal number of clusters (as implemented in PACK) is unlikely to capture structure because of a large false negative rate [39]. Hence, in order to define groups of over-expression and under-expression, we applied the pam algorithm with two centers to each of the seven genes in each of the two cohorts (Figure 4 and Additional data files 6 and 7). Because the small number of events, six, in each of the two external cohorts implied a highly discrete $P$ value distribution, Fisher test $P$ values would be too conservative and poor approximations for type I errors [40]. To overcome this difficulty, we also considered the distribution of good and poor outcome samples over the combined clusters of over-expression and under-expression (Table 5). This showed that four of the seven genes (C1QA, IGLC2, TNFRSF 17 , and $L Y 9$ ) were also highly prognostic in these two external cohorts, thus confirming the validity of our finding. Moreover, as in the three original cohorts, over-expression of these genes in the two external cohorts was associated with good prognosis (Figure 4 and Additional data files 6 and 7). For the other three genes (HLA-F, SPP1, and XCL2), $P$ values did not reach statistical significance ( $P$ about 0.2$)$, yet their expression profile trends were entirely consistent with those found in the integrated cohort, thus confirming their role as members of a robust prognostic module (Additional data files 6 and 7).
To confirm the robustness of our findings, we used the pam algorithm to classify patients in the two external cohorts into clusters over-expressing and under-expressing the IR module (Figure 5a,b). Remarkably, the predicted 20-sample cluster over-expressing the module was composed entirely of good outcome patients, whereas the remaining 35 -sample cluster included 12 poor outcome events (Figure $5 \mathrm{c}$ ), which was highly significant under Fisher's exact test $(P=0.002$; the HR is singular because one cluster did not have any events).

\section{The prognostic value of the IR module is specific to ER- tumours}

To confirm that the good prognosis conferred by activation of the IR module is specific to ER-breast cancer, we applied the same pam clustering algorithm over the seven genes to the integrated dataset of $527 \mathrm{ER}^{+}$breast tumors. This gave two clusters with unequal distributions of good and poor outcome samples (209 good and 99 poor for the cluster under-expressing the genes versus 163 good and 47 poor for the cluster overexpressing the genes; $P=0.02$ ). Although this suggested to us that over-expression of this seven-gene module also conferred better prognosis in $\mathrm{ER}^{+}$samples, the association was much weaker than for ER-samples. Univariate Cox regression with TTDM as the outcome variable confirmed that underexpression of this seven-gene module conferred a much greater risk for distant metastasis in ER- tumors (HR 2.02, 95\% CI 1.2 to $3.4 ; P=0.009$ ) than in $\mathrm{ER}^{+}$tumors (HR 1.25, $95 \% \mathrm{CI} 0.9$ to 1.7 ; $P=0.16$; Table 4 ). It is also noteworthy that, in contrast to the ER- case, in the multivariate model setting for $\mathrm{ER}^{+}$tumors, a low LI score and LN involvement were stronger predictors of TTDM than the seven-gene module (HR 1.65, 95\% CI 0.4 to 7.1 for LI score and HR 1.48, 95\% CI 0.7 to 3.2 for LN involvement, versus HR $<1$; Table 4). The specificity of our prognostic module to ER-breast cancer was 
Table 5

\begin{tabular}{|c|c|c|c|c|c|c|c|c|c|c|}
\hline \multirow[t]{2}{*}{ Gene } & & \multicolumn{3}{|c|}{ UPP } & \multicolumn{3}{|c|}{$\mathrm{JRH}-2$} & \multicolumn{3}{|c|}{ Combined } \\
\hline & & Poor & Good & $P$ & Poor & Good & $P$ & Poor & Good & $P$ \\
\hline \multirow[t]{2}{*}{ IGLC2 } & High & 0 & 12 & 0.04 & 0 & 7 & 0.09 & 0 & 19 & 0.003 \\
\hline & Low & 6 & 13 & & 6 & II & & 12 & 24 & \\
\hline \multirow[t]{2}{*}{ LY9 } & High & 1 & 16 & 0.05 & 0 & 6 & 0.14 & I & 22 & 0.007 \\
\hline & Low & 5 & 9 & & 6 & 12 & & II & 21 & \\
\hline \multirow[t]{2}{*}{ TNFRSFI7 } & High & I & 16 & 0.05 & 0 & 10 & 0.02 & I & 26 & 0.001 \\
\hline & Low & 5 & 9 & & 6 & 8 & & II & 17 & \\
\hline \multirow[t]{2}{*}{ CIQA } & High & 0 & 12 & 0.04 & 0 & 9 & 0.04 & 0 & 21 & 0.001 \\
\hline & Low & 6 & 13 & & 6 & 9 & & 12 & 22 & \\
\hline
\end{tabular}

confirmed by application of PAC, which showed that, with the exception of $X C L 2$, none of the other six genes were individually prognostic.

In this context, it is worth noting once again the absence of immune response related genes among the 29 PAC derived prognostic genes in $\mathrm{ER}^{+}$disease, which would suggest that a good prognosis IR related subtype is absent in $\mathrm{ER}^{+}$breast cancer. To investigate this further we checked, by performing Wilcoxon rank sum tests on the 5,007 genes, that the absence of immune response GOs was not just an artefact of the small number of genes picked out by PACK. In fact, GO analysis (using GOTree Machine) of the top 500 genes obtained from this rank sum test (all with q-values < 0.05) showed that cell cycle and transcription regulator activity related GOs were the only categories with highly significant $P$ values (uncorrected $P<10^{-6}$ ), and that the only enriched immune response related GO was that of humoral immune response, with 11 represented genes (including $B L M, F A D D, C_{3}, C_{7}, B C L 2$, NFKB1, and the IR module member TNFRSF17), and which was only marginally enriched (uncorrected $P=0.005$ ). Although we verified that this humoral immune response module was associated with prognosis in the integrated $\mathrm{ER}^{+}$ cohort independent of LN status and LI (HR 2.26, 95\% CI 0.97 to $5.26 ; P=0.06$ ) we were unable to validate this prognostic module in the external UPP and JRH-2 cohorts (Additional data file 8). Moreover, the co-regulation patterns for the genes in this module were less coherent than those for the IR module in ER- breast cancer (Figure 5 and Additional data file 8). Hence, independent of the methodology used, an IR related prognostic module in $\mathrm{ER}^{+}$breast cancer could not be identified, which seems to suggest that a good prognosis subtype related to IR is specific to ER- disease.

\section{Discussion}

A striking difference between $\mathrm{ER}^{+}$and $\mathrm{ER}^{-}$disease is emerging at the level of mRNA expression. Although in $\mathrm{ER}^{+}$disease a significant number of genes have been found that correlate with clinical outcome $[5,10,18,22]$, in ER- disease no such prognostic signatures have thus far been reported. Moreover, although in $\mathrm{ER}^{+}$tumors subtypes of different prognostic risks, the luminal $\mathrm{A}$ and $\mathrm{B}$ subtypes, have been defined [21,22], no such subdivisions have been noted for ER- breast cancer. It is known that the two main subtypes of ER-breast cancer (ER-/HER2 ${ }^{+}$and basals) have worse prognosis compared with the luminal A subtype, but no outcome differences between the ER-/HER2 ${ }^{+}$and basal subtypes have been observed $[15,21-23,26]$.

We believe that these differences between $\mathrm{ER}^{+}$and $\mathrm{ER}^{-}$disease are related to the different histopathologic characteris-

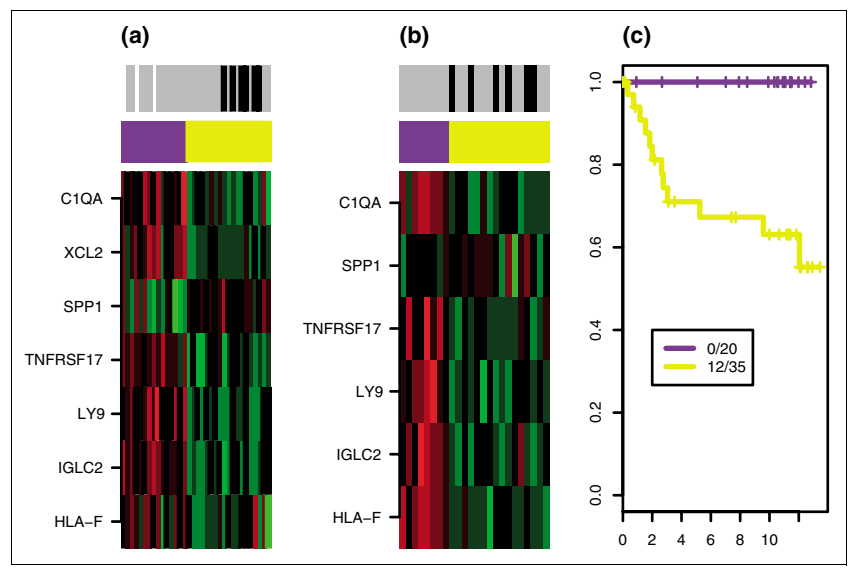

Figure 5

Pam clustering over IR module in external ER- cohorts. Heatmap of gene expression of seven-gene IR-module in ER- samples of the (a) UPP and (b) JRH-2 cohorts. Shown are the clusters over-expressing (purple) and under-expressing (yellow) the IR module, as predicted by the pam algorithm. Good outcome samples are shown in gray, and poor outcome samples in black. Green indicates relative under-expression, and red indicates relative over-expression. (c) Kaplan-Meier survival curves over combined external cohorts (for UPP end-point was disease-specific survival, and for JRH-2 it was recurrence-free survival), with the number of events and samples in each of the two predicted groups. ER, estrogen receptor; pam, partitioning around medoids. 
tics of the tumors. The prognostic signatures derived for $\mathrm{ER}^{+}$ breast cancer are characterized by genes related to cell cycle and cell proliferation pathways, and are also highly correlated with the histologic grade of the tumors $[7,22]$. It is not a coincidence that most luminal $\mathrm{B}$ tumours are of high grade, whereas the great majority of luminal A tumours are of low grade $[7,22]$. It appears that there may be a whole plethora of diverse oncogenic pathways that drive the over-activation of cell cycle and cell growth pathways in poor prognosis tumors. This would explain the larger number of prognostic genes found in $\mathrm{ER}^{+}$disease (the great majority of which are related to cell cycle ontologies) as well as the stronger prognostic signals (relatively large differences in log2 expression between poor and good prognosis tumours), an effect that is probably driven by oncogenic amplifications. This interpretation would also fit in well with our finding that most bimodal profiles in $\mathrm{ER}^{+}$breast cancer have positive kurtosis values, because this could be a reflection of a more diverse range of small amplifier subgroups in $\mathrm{ER}^{+}$breast cancer.

In contrast, most ER-tumours are of high grade, which would explain why any differences in clinical outcome within ERdisease are not related to differential activation of cell cycle pathways. Instead, the work presented here shows that differences in clinical outcome within ER- disease are mainly related to differentially expressed genes in the complement and immune response pathways, and that the association with prognosis can be independent of lymphocytic infiltration (LI) and LN status. In fact, for ER- tumors we observed that even though there were proportionally more high LI samples in the group over-expressing the IR module, these did not necessarily have better prognosis. The fact that LI could not explain the observed association of the IR module with outcome was supported further by our finding that medullary breast cancers, which are characterized by high LI scores, had expression profiles most similar to the $\mathrm{CC}^{+} / \mathrm{IR}^{+}$subtype rather than the $\mathrm{IR}^{+}$subtype, which had the best prognosis overall. The better prognosis of the $\mathrm{CC}^{+} / \mathrm{IR}^{+}$subtype relative to $\mathrm{CC}^{+}$and $\mathrm{SR}^{+} \mathrm{ER}^{-}$breast cancer is therefore entirely consistent with the $\mathrm{CC}^{+} / \mathrm{IR}^{+}$subclass being medullary breast cancers (MBCs), as MBC is known to have marginally better prognosis than other basal tumors [38]. On the other hand, the $\mathrm{IR}^{+}$subclass, which had the best prognosis among the five ER- subclasses, was only marginally associated with high LI and was unrelated to MBC. Also consistent with these observations, it is important to note again the distinction between the identified seven-gene prognostic IR module and the 98gene IR cluster that was derived from unsupervised hierarchical clustering. Clearly, we found a strong statistical association between high LI and over-expression of the 98-gene IR cluster. Specifically, there were 13 high LI and eight low LI samples in the combined $\mathrm{IR}^{+}$and $\mathrm{CC}^{+} / \mathrm{IR}^{+}$clusters relative to six high LI and 23 low LI samples in the rest of the cohort (Fisher test $P=0.007$ ). In contrast, the association between high LI and over-expression of the IR module was much weaker $(P=0.07)$. Again, this suggests that a significant number of genes in the 98-gene IR cluster show expression variability that is not explained by LI. This is confirmed further by two recent studies that profiled breast cancer cell lines [27,38], which showed that a considerable number of immune response related genes do exhibit significant variable expression across the basal cell subtype. Moreover, we found that two (SPP1 and $H L A-F$ ) of the three IR module genes that we could map to good quality probes in $[27,38]$ showed twofold changes across the eight basal cell lines.

Thus, these findings together suggest that a significant proportion of the expression of the IR module genes in the good prognosis tumors is tumor-intrinsic in origin. That tumorintrinsic expression of IR genes can have an impact on prognosis of breast cancer patients is plausible in view of recent studies that show, for example, how amplification of kinase oncogenes can activate the nuclear factor- $\kappa \mathrm{B}$ pathway, and hence immune response pathways, in both breast cancer cell lines and patient derived breast tumors [41]. Similarly, another recent study [42] used breast cancer cell lines to show how BRCA1/IFN- $\gamma$ pathways may regulate target genes involved in innate immune response, providing another possible mechanism for tumor intrinsic IR gene expression variability.

In spite of identifying only a relatively small module of prognostic genes, we were nevertheless able to validate their prognostic potential in two external cohorts. It is likely that an integrative analysis similar to the one used here but applied to multiple cohorts that were all profiled on exactly the same genome-wide platform would allow further expansion of this module to include other members of the complement and immune response pathways. Interestingly, from the seven prognostic markers that composed the IR module, two have already been associated with clinical outcome in breast cancer. Specifically, C1QA, which is a gene involved in the classical complement pathway, was recently shown to harbor a single nucleotide polymorphism that correlated with distant metastasis in breast cancer [43]. Two recent studies also implicated $S P P 1$ (osteopontin) in metastatic breast cancer [44,45].

It is important also to note that the robustness of the identified prognostic markers is a consequence of the PACK methodology. Despite being a conservative procedure that filters out many true positives, PACK allows, by efficient removal of false positives, a more reliable identification of prognostic markers. We tested this further by applying two popular statistical tools, singular value decomposition (SVD) [46] and the shrunken centroids classifier (PAMR) [47], to the integrated ER- dataset to determine whether we could derive a similar if not identical prognostic IR module. Using SVD we found that none of the inferred SVD components showed a correlation with prognosis (Wilcoxon rank sum test $P>0.05$ ), whereas at a FDR threshold of 0.3 PAMR yielded 21 prognostic genes, of which only two (the IR module member 
TNFRSF17 and KLRD1) had immune response related functions. Thus, in agreement with findings presented previously [17], this reinforces the advantage of PACK over other pattern recognition tools and supervised methods that do not use pattern recognition steps, such as for example those based on $t$-tests.

The absence of a prognostic IR module in $\mathrm{ER}^{+}$breast cancer is intriguing. The seven-gene IR module was only marginally associated with prognosis in $\mathrm{ER}^{+}$disease, and importantly this association was not independent of LI or LN status. Repeating the same unsupervised analysis (PAK) and semisupervised analysis (PACK) in ER+ breast cancer also did not find a prognostic immune response module. By using a traditional supervised method, a prognostic IR module was identifiable but failed to validate in the two external cohorts. Thus, to determine fully whether such a robust prognostic IR module exists for $\mathrm{ER}^{+}$breast cancer, it may be necessary to conduct larger integrative studies that use the same microarray platform so that the analysis can be performed over a larger set of common genes.

Besides identifying a module of genes that is prognostic in over 240 ER- breast tumors, PACK also provided us with a novel subclassification of ER-breast cancer. Specifically, clustering over PACK selected genes identified five different subtypes $\left(\mathrm{CC}^{+}, \mathrm{CC}^{+} / \mathrm{IR}^{+}, \mathrm{IR}^{+}, \mathrm{ECM}^{+}\right.$, and $\left.\mathrm{SR}^{+}\right)$characterized by the over-expression patterns of four distinct gene clusters, each enriched for IR, ECM, CC, and SR genes, respectively. Moreover, we related these subtypes to the gene expression based intrinsic subclasses. This showed that the basal subgroup was a heterogeneous group with at least four distinct subtypes $\left(\mathrm{CC}^{+}, \mathrm{CC}^{+} / \mathrm{IR}^{+}, \mathrm{ECM}^{+}\right.$, and $\left.\mathrm{IR}^{+}\right)$, whereas the $\mathrm{ER}^{-} /$ $\mathrm{HER2}^{+}$subgroup showed strong overlap with the $\mathrm{SR}^{+}$and $\mathrm{IR}^{+}$ subtypes.

\section{Conclusion}

While in $\mathrm{ER}^{+}$breast cancer prognostic markers are associated mainly with cell cycle pathways, in ER- disease prognostic markers are associated with immune response pathways. In particular, we have identified a subclass of ER- tumors that over-express immune response genes and that has a good prognosis compared with the rest of ER-breast tumors, independently of LN status or LI. Furthermore, we have identified an associated module of complement and immune response genes that define prognostic markers valid in over $240 \mathrm{ER}^{-}$ samples.

\section{Materials and methods Datasets and gene annotation}

The microarray breast cancer datasets considered in this work are described elsewhere [5,7,9,18,19]. For these cohorts we used the normalized data, which are available in the public domain (see $[5,7,9,18,19]$ ). The retrieved datasets were fur- ther normalized, if necessary, by transforming them onto a common log2 scale and shifting the median of each array to zero. We also created an automated computational pipeline (Perl scripts on a Linux platform) to crosslink the annotation provided for each dataset with UniGene. For some datasets, the linkage relied on Ensembl [48] external database identifiers. Thus each probe was associated with a universal gene name. This procedure generated a nonredundant set of gene identifiers for the subsequent integrative analysis.

\section{PACK: profile analysis using clustering and kurtosis}

The hypothesis underlying PACK [17] is that genes that are true biologic or clinical markers have expression profiles that are generated by a mixture of two or more underlying distributions, whereas spurious features are more likely to have profiles generated by a single distribution. The biologic validity of this assumption was proved through a FDR analysis [17].

PACK can be viewed as a semi-supervised algorithm, consisting of two main steps: a feature selection criterion and a supervised step, in which the selected features are correlated to a phenotype (Figure 2). It is important to note that PACK is a flexible modular algorithm in that the feature selection step can be applied on its own. In this case, there are two possible versions of the algorithm: PAC and PAK. The precise way in which these two algorithms are used in PACK will depend on the purpose of the exercise. Below, we describe the PACK strategy implemented in this paper, which is slightly different from that applied previously [17].

\section{Feature selection with PAK: using negative kurtosis to find genes defining major subclasses}

Kurtosis is related to the fourth central moment and can conveniently be defined as follows [49]:

$$
K(X) \equiv \frac{\mathrm{E}\left[(\grave{\mathrm{e}}-\overline{\mathrm{e}})^{4}\right]}{\mathrm{E}\left[(\grave{\mathrm{e}}-\overline{\mathrm{e}})^{2}\right]^{2}}-3
$$

where $\mathrm{X}$ is any random variable and $\mathrm{E}$ denotes the expectation. For a gaussian $\mathrm{E}\left[(\grave{\mathrm{e}}-\overline{\mathrm{e}})^{4}\right]=3 \mathrm{E}\left[(\grave{\mathrm{e}}-\overline{\mathrm{e}})^{2}\right]^{2}$, so that $\mathrm{K}(\mathrm{X})=0$. Most nongaussian distributions necessarily have either $\mathrm{K}>\mathrm{o}$, in which case they are called supergaussian or leptokurtic, or $\mathrm{K}<\mathrm{O}$, in which case they are called subgaussian or platykurtic. Specifically, a mixture of two approximately equal mass gaussians must have negative kurtosis because the two modes on either side of the center of mass effectively flatten out the distribution. To see this, consider a gene whose expression profile is described by a mixture of two gaussians. Then, the kurtosis, $\mathrm{K}$, is a function of two parameters (we assume for simplicity that the gaussians are of equal variance $\sigma^{2}$, although this assumption is not needed for the result below); the effect size of the gene, as defined by the effective separation e between the two gaussians $(\mathrm{e}=\mu / \sigma$, where $\mu$ is the separation), and the ratio of cluster weights $\left(\pi_{1}\right.$, 
$\left.\pi_{2}\right)$, that is $\mathrm{R}=\pi_{1} /\left(1-\pi_{1}\right)$. Specifically, a short computation reveals that

$$
K(e, R)=e^{4} \frac{R(R-a)(R-b)}{(1+R)^{4}\left(1+\frac{R}{(1+R)^{2}} e^{2}\right)^{2}}
$$

where $\mathrm{a}$ and $\mathrm{b}$ are the quadratic roots $2 \pm \sqrt{3}$. Thus, for $\mathrm{e} \neq \mathrm{O}$, the kurtosis is negative if and only if $(2-\sqrt{3})<R<(2+\sqrt{3})$. This in turn requires the smallest cluster weight, $\pi_{\text {min }}$, to be in the range (approximately) of $0.22<\pi_{\min }<0.5$. It follows that for the case of approximately equal weights, where $\mathrm{R} \cong 1\left(\pi_{\min }\right.$ $\cong 0.5$ ), the kurtosis is always negative and in the limit of large cluster separations (when e >> 1) the kurtosis decreases monotonically, asymptotically approaching the lower bound 2. Thus, kurtosis provides a useful measure for ranking genes based on how platykurtic their profiles are.

Given a gene's expression profile $\mathrm{x}=\left(\mathrm{x}_{1}, \ldots, \mathrm{x}_{\mathrm{n}}\right)$, an unbiased estimate for the kurtosis [50] is as follows:

$$
K(x) \triangleq \frac{n(n+1) \sum_{i=1}^{n}\left(x_{i}-\bar{x}\right)^{4}}{(n-1)(n-2)(n-3) \sigma^{4}}-\frac{3(n-1)^{2}}{(n-2)(n-3)}
$$

where $\bar{x}$ and $\sigma$ are the mean and standard deviation estimates of the profile. A standard error estimate of $\mathrm{K}$ was obtained by performing 10,000 random simulations, with $n=$ 186 (number of ER- samples), which showed that the standard error estimate, 0.36 , was essentially identical to the theoretical estimate, $\sqrt{ }(24 / n)$ [50].

Two notes with the feature selection step are in order. First, the kurtosis threshold used to select features depends on how large the smallest subgroup must be. Generally, given the effective separation values that are typical for differential gene expression, we find that a zero kurtosis threshold (as used in this report) generally picks out subgroups within the individual gene expression profiles that are at least as large as $30 \%$ of the total sample size [17]. Second, in principle, genes defining major subclasses could be found using a clustering step to infer two clusters (PAC) and setting a lower bound threshold (for instance, 30\%) on the size of the smallest cluster. However, this approach is computationally more expensive, because PAC attempts to estimate the optimal number of clusters in the profile. However, this model selection step is a necessary one to ensure that profiles for which there is no objective evidence of bimodality are excluded (see below).

\section{PAC: identification of robust prognostic markers}

Having selected the genes defining the largest subclasses, we next apply PAC to each of these genes to remove those for which there is no evidence of bimodality (gaussian profiles that spuriously have negative kurtosis values). Specifically, given a gene's expression profile $\mathrm{x}=\left(\mathrm{x}_{1}, \ldots, \mathrm{x}_{\mathrm{n}}\right)$, we model this as a random sample of a univariate random variable $\mathrm{X}$, whose density function is possibly a mixture of Gaussians:

$$
p\left(x_{i} \mid \theta\right)=\sum_{k=1}^{C_{M}} \pi_{k} G\left(x_{i} \mid \mu_{k}, \sigma_{k}\right)
$$

Where $\pi_{\mathrm{k}}$ are the weights of the components, $\left(\mu_{\mathrm{k}}, \sigma_{\mathrm{k}}\right)$ are the mean and standard deviation of the univariate gaussian $\mathrm{k}$, and $\theta$ denotes the set of all parameters. In the above, $\mathrm{C}_{\mathrm{M}}$ denotes the maximum number of clusters that can be inferred, which in our application we set to 2. The optimal number of clusters, $\mathrm{C}$, can be inferred using one of various approaches. One possibility is to use the EM algorithm to learn the parameters for the two different models $\mathrm{C}=1$ and $\mathrm{C}$ $=2$, and perform model selection using the Bayesian Information Criterion (BIC) score [51,52]. Alternatively, the optimal number of clusters, $\mathrm{C}$, can be inferred using a lower bound on the model evidence, as provided by a variational Bayesian (VB) approach [39,53,54]. The results we report here were obtained using the VB algorithm for model selection. Thus, genes for which $\mathrm{C}=1$ were excluded from further analysis. Finally, association with the phenotype (here prognosis) was determined using Fisher's exact test to test whether poor outcome events were unevenly distributed across the two clusters.

\section{Software packages used}

All analyses were performed using the R statistical programming language [55]. The following add-on packages were used: vabayelMix for the PACK implementation, survival for the Cox regression models, qvalue for FDR estimation, and cluster for the partitioning around medoids (pam) clustering algorithm.

\section{The SSP classifier}

The classification of the samples in the NKI2, EMC, and $\mathrm{NCH}$ cohorts into the intrinsic subtypes was performed using the single sample predictor (SSP) [23] and was done for each cohort separately because this guaranteed a larger number of overlapping genes. In the SSP, samples were assigned the intrinsic subtype for which the corresponding Spearman rank correlation between the sample and SSP centroid was maximal [23].

\section{The ER- subclass centroids}

From the hierarchical clustering with Pearson correlation metric and complete linkage diagram (Figure 3a) we constructed mean centroids for each of the five subclasses. Classification of external samples to these centroids was performed using the nearest centroid criterion. Because these centroids were defined over ER- samples only, external samples (which may not be ER-) may not show strong correlation to any of these centroids. We thus validated, through 10,000 Monte Carlo (MC) randomisations, that samples with a maximal pearson coefficient larger than 0.25 were significantly correlated with the corresponding centroid $(P<0.0001)$. Samples with maximal correlation coefficients smaller than 0.25 were deemed to be unclassifiable. 


\section{Expression based basal and HER2 ${ }^{+}$markers}

The basal marker used in Figure 3 was derived by first mapping ten validated basal markers (CRYAB, ANXA8, LAMC2, LAMB3, ITGA6, KRT17, KRT15, KRT13, KRT6B, and KRT5) [27] onto the integrated data set of 5,007 genes. For each of these markers samples were ranked in order of decreasing expression or 'basalness'. For each sample in the integrated cohort an average rank was then computed over the ten basal markers. The average ranks were then rescaled onto the unit interval $(0,1)$, with ' 1 ' indicating highest expression for basal markers. The marker for the ERBB2 subtype was obtained in an analogous manner using three genes in the ERBB2 amplicon (ERBB2, GRB7, and STARD3).

\section{Lymphocyte infiltration scores}

For the samples from our $\mathrm{NCH}$ cohort [9] we used the following scoring method. Lymphocytic infiltration (LI) was assessed in whole tumour sections from frozen sections stained with hematoxylin and eosin. The intensity of lymphocytic infiltrate was first graded semi-quantitatively as minimal or mild (1), moderate (2), and marked (3). The LI scores were then dichotomized (we considered mild and moderate as low LI and marked as high LI) to make them comparable with the binary LI scores used by van 't Veer and coworkers [3].

\section{Additional data files}

The following additional data are available with the online version of this manuscript. Additional data file 1 is a table showing the 813 genes with negative kurtosis expression profiles over 186 ER- tumors, together with the predicted number of clusters and Fisher's test $P$ value with outcome as binary phenotype. Additional data file 2 is a table showing the 193 genes with negative kurtosis expression profiles over 527 ER+ samples, together with the predicted number of clusters and Fisher's test $P$ value with outcome as binary phenotype. Additional data file 3 is a figure showing hierarchical clustering over 186 ER- breast cancers (gene annotated version). Additional data file 4 is a figure showing the distribution of basal and ERBB2 markers among ER- subtypes. Additional data file 5 is a table showing the centroids of gene expression for each of the five identified ER- subtypes. Additional data file 6 is a figure showing expression profiles of immune response module genes in ER- samples of the external UPP cohort. Additional data file 7 is a figure showing expression profiles of immune response module genes in ER- samples of the external JRH-2 cohort. Additional data file 8 is a figure showing the clustering of ER+ samples over the humoral immune response gene module in the two external UPP and JRH-2 cohorts.

\section{Acknowledgements}

This research was supported by grants from Cancer Research UK. We thank Ali Naderi for useful discussions.

\section{References}

I. Brenton JD, Carey LA, Ahmed AA, Caldas C: Molecular classification and molecular forecasting of breast cancer: ready for clinical application? J Clin Oncol 2005, 23:7350-7360.

2. Rakha EA, El-Sayed ME, Green AR, Paish EC, Lee AH, Ellis IO: Breast carcinoma with basal differentiation: a proposal for pathology definition based on basal cytokeratin expression. Histopathology 2007, 50:434-438.

3. van 't Veer LJ, Dai $H$, van de Vijver MJ, He YD, Hart AA, Mao M, Peterse HL, van der Kooy K, Marton MJ, Witteveen AT, et al.: Gene expression profiling predicts clinical outcome of breast cancer. Nature 2002, 4I5:530-536.

4. Paik S, Shak S, Tang G, Kim C, Baker J, Cronin M, Baehner FL, Walker MG, Watson D, Park T, et al.: A multigene assay to predict recurrence of tamoxifen-treated, node-negative breast cancer. N Engl J Med 2004, 35 I:28I7-2826.

5. Wang Y, Klijn JG, Zhang Y, Sieuwerts AM, Look MP, Yang F, Talantov $D$, Timmermans M, Meijer-van Gelder ME, Yu J, et al:: Gene-expression profiles to predict distant metastasis of lymph-nodenegative primary breast cancer. Lancet 2005, 365:67I-679.

6. Pawitan Y, Bjohle J, Amler L, Borg AL, Egyhazi S, Hall P, Han X, Holmberg L, Huang F, Klaar S, et al.: Gene expression profiling spares early breast cancer patients from adjuvant therapy: derived and validated in two population-based cohorts. Breast Cancer Res 2005, 7:R953-R964.

7. Sotiriou C, Wirapati P, Loi S, Harris A, Fox S, Smeds J, Nordgren H, Farmer P, Praz V, Haibe-Kains B, et al.: Gene expression profiling in breast cancer: understanding the molecular basis of histologic grade to improve prognosis. J Natl Cancer Inst 2006, 98:262-272.

8. Foekens JA, Atkins D, Zhang Y, Sweep FC, Harbeck N, Paradiso A, Cufer T, Sieuwerts AM, Talantov D, Span PN, et al.: Multicenter validation of a gene expression-based prognostic signature in lymph node-negative primary breast cancer. J Clin Oncol 2006, 24:1665-167I.

9. Naderi A, Teschendorff AE, Barbosa-Morais NL, Pinder SE, Green AR, Powe DG, Robertson JF, Aparicio S, Ellis IO, Brenton JD, et al.: A gene-expression signature to predict survival in breast cancer across independent data sets. Oncogene 2007, 26: $1507-1516$.

10. Teschendorff AE, Naderi A, Barbosa-Morais NL, Pinder SE, Ellis IO, Aparicio S, Brenton JD, Caldas C: A consensus prognostic gene expression classifier for ER positive breast cancer. Genome Biol 2006, 7:RIOI.

II. van de Rijn M, Perou CM, Tibshirani R, Haas P, Kallioniemi $O$, Kononen J, Torhorst J, Sauter G, Zuber M, Kochli OR, et al.: Expression of cytokeratins 17 and 5 identifies a group of breast carcinomas with poor clinical outcome. Am J Pathol 2002, 161:1991-1996.

12. Malzahn K, Mitze M, Thoenes M, Moll R: Biological and prognostic significance of stratified epithelial cytokeratins in infiltrating ductal breast carcinomas. Virchows Arch 1998, 433:I I9-I29.

13. Rakha EA, El-Rehim DA, Paish C, Green AR, Lee AH, Robertson JF, Blamey RW, Macmillan D, Ellis IO: Basal phenotype identifies a poor prognostic subgroup of breast cancer of clinical importance. Eur J Cancer 2006, 42:3|49-3I56.

14. Rakha EA, El-Sayed ME, Green AR, Lee AH, Robertson JF, Ellis IO: Prognostic markers in triple-negative breast cancer. Cancer 2007, 109:25-32.

15. Jumppanen M, Gruvberger-Saal S, Kauraniemi P, Tanner M, Bendahl PO, Lundin M, Krogh M, Kataja P, Borg A, Ferno M, et al.: Basal-like phenotype is not associated with patient survival in estrogen-receptor-negative breast cancers. Breast Cancer Res 2007, 9:RI6.

16. Eden P, Ritz C, Rose C, Ferno M, Peterson C: 'Good Old' clinical markers have similar power in breast cancer prognosis as microarray gene expression profilers. Eur J Cancer 2004, 40:|837-184I.

17. Teschendorff AE, Naderi A, Barbosa-Morais NL, Caldas C: PACK: Profile Analysis using Clustering and Kurtosis to find molecular classifiers in cancer. Bioinformatics 2006, 22:2269-2275.

18. van de Vijver MJ, He YD, van't Veer LJ, Dai H, Hart AA, Voskuil DW, Schreiber GJ, Peterse JL, Roberts C, Marton MJ, et al.: A geneexpression signature as a predictor of survival in breast cancer. N Engl J Med 2002, 347:1999-2009.

19. Miller LD, Smeds J, George J, Vega VB, Vergara L, Ploner A, Pawitan $Y$, Hall P, Klaar S, Liu ET, Bergh J: An expression signature for p53 
status in human breast cancer predicts mutation status, transcriptional effects, and patient survival. Proc Natl Acad Sci USA 2005, 102:13550-13555.

20. Storey JD, Tibshirani R: Statistical significance for genomewide studies. Proc Natl Acad Sci USA 2003, I 00:9440-9445.

21. Sorlie T, Tibshirani R, Parker J, Hastie T, Marron JS, Nobel A, Deng S, Johnsen H, Pesich R, Geisler S, et al.: Repeated observation of breast tumor subtypes in independent gene expression data sets. Proc Natl Acad Sci USA 2003, 100:84 I8-8423.

22. Sotiriou C, Neo SY, McShane LM, Korn EL, Long PM, Jazaeri A, Martiat P, Fox SB, Harris AL, Liu ET: Breast cancer classification and prognosis based on gene expression profiles from a population-based study. Proc Natl Acad Sci USA 2003, 100:10393-10398.

23. Hu Z, Fan C, Oh DS, Marron JS, He X, Qaqish BF, Livasy C, Carey LA, Reynolds E, Dressler L, et al:: The molecular portraits of breast tumors are conserved across microarray platforms. $B M C$ Genomics 2006, 7:96.

24. Farmer $\mathrm{P}$, Bonnefoi $\mathrm{H}$, Becette $\mathrm{V}$, Tubiana-Hulin $\mathrm{M}$, Fumoleau $\mathrm{P}$, Larsimont D, Macgrogan G, Bergh J, Cameron D, Goldstein D, et al.: Identification of molecular apocrine breast tumours by microarray analysis. Oncogene 2005, 24:4660-467I.

25. Doane AS, Danso M, Lal P, Donaton M, Zhang L, Hudis C, Gerald WL: An estrogen receptor-negative breast cancer subset characterized by a hormonally regulated transcriptional program and response to androgen. Oncogene 2006, 25:3994-4008.

26. Perou CM, Sorlie T, Eisen MB, van de Rijn M, Jeffrey SS, Rees CA, Pollack JR, Ross DT, Johnsen H, Akslen LA, et al.: Molecular portraits of human breast tumours. Nature 2000, 406:747-752.

27. Charafe-Jauffret E, Ginestier C, Monville F, Finetti P, Adelaide J, Cervera N, Fekairi S, Xerri L, Jacquemier J, Birnbaum D, Bertucci F: Gene expression profiling of breast cell lines identifies potential new basal markers. Oncogene 2006, 25:2273-2284.

28. Zhang B, Schmoyer D, Kirov S, Snoddy J: GOTree Machine (GOTM): a web-based platform for interpreting sets of interesting genes using Gene Ontology hierarchies. BMC Bioinformatics 2004, 5: I-8.

29. Kaufman L, Rousseeuw PJ: Finding Groups in Data: An Introduction to Cluster Analysis New York: Wiley; 1990.

30. Huang E, Cheng SH, Dressman H, Pittman J, Tsou MH, Horng CF, Bild $A$, Iversen ES, Liao $M$, Chen $C M$, et al.: Gene expression predictors of breast cancer outcomes. Lancet 2003, 36 I:1590-1596.

31. Lee AH, Gillett CE, Ryder K, Fentiman IS, Miles DW, Millis RR: Different patterns of inflammation and prognosis in invasive carcinoma of the breast. Histopathology 2006, 48:692-70I.

32. Marques LA, Franco EL, Torloni $H$, Brentani MM, da Silva-Neto JB, Brentani RR: Independent prognostic value of laminin receptor expression in breast cancer survival. Cancer Res 1990, 50: |479-| 483 .

33. Nixon AJ, Neuberg D, Hayes DF, Gelman R, Connolly JL, Schnitt S, Abner A, Recht A, Vicini F, Harris JR: Relationship of patient age to pathologic features of the tumor and prognosis for patients with stage I or II breast cancer. J Clin Oncol 1994, 1 2:888-894.

34. Rilke F, Colnaghi MI, Cascinelli N, Andreola S, Baldini MT, Bufalino R, Della Porta G, Menard S, Pierotti MA, Testori A: Prognostic significance of HER-2/neu expression in breast cancer and its relationship to other prognostic factors. Int J Cancer I99I, 49:44-49.

35. Aaltomaa S, Lipponen P, Eskelinen M, Kosma VM, Marin S, Alhava E, Syrjanen K: Lymphocyte infiltrates as a prognostic variable in female breast cancer. Eur J Cancer 1992, 28A:859-864.

36. Holmberg L, Adami HO, Lindgren A, Ekbom A, Sandstrom A, Bergstrom R: Prognostic significance of the Ackerman classification and other histopathological characteristics in breast cancer. An analysis of $\mathrm{I}, 349$ consecutive cases with complete follow-up over seven years. APMIS 1988, 96:979-990.

37. Carlomagno C, Perrone F, Lauria R, de Laurentiis M, Gallo C, Morabito A, Pettinato G, Panico L, Bellelli T, Apicella A, et al.: Prognostic significance of necrosis, elastosis, fibrosis and inflammatory cell reaction in operable breast cancer. Oncology 1995, 52:272-277.

38. Bertucci F, Finetti P, Cervera N, Charafe-Jauffret E, Mamessier E, Adelaide J, Debono S, Houvenaeghel G, Maraninchi D, Viens P, et al.: Gene expression profiling shows medullary breast cancer is a subgroup of basal breast cancers. Cancer Res 2006, 66:4636-4644.

39. Teschendorff AE, Wang Y, Barbosa-Morais NL, Brenton JD, Caldas C: A variational Bayesian mixture modelling framework for cluster analysis of gene-expression data. Bioinformatics 2005
2I:3025-3033.

40. Agresti A: Categorical Data Analysis. Wiley Series in Probability and Statistics New York: Wiley; 2002.

4I. Boehm JS, Zhao J], Yao J, Kim SY, Firestein R, Dunn IF, Sjostrom SK, Garraway LA, Weremowicz S, Richardson AL, et al.: Integrative genomic approaches identify IKBKE as a breast cancer oncogene. Cell 2007, I 29:1065-1079.

42. Buckley NE, Hosey AM, Gorski JJ, Purcell JW, Mulligan JM, Harkin DP, Mullan PB: BRCAI regulates IFN-gamma signaling through a mechanism involving the type I IFNs. Mol Cancer Res 2007, 5:26I-270.

43. Racila E, Racila DM, Ritchie JM, Taylor C, Dahle C, Weiner G]: The pattern of clinical breast cancer metastasis correlates with a single nucleotide polymorphism in the CIqA component of complement. Immunogenetics 2006, 58: I-8.

44. Allan AL, George R, Vantyghem SA, Lee MW, Hodgson NC, Engel CJ, Holliday RL, Girvan DP, Scott LA, Postenka CO, et al:: Role of the integrin-binding protein osteopontin in lymphatic metastasis of breast cancer. Am J Pathol 2006, 169:233-246.

45. de Silva Rudland S, Martin L, Roshanlall C, Winstanley J, Leinster S, Platt-Higgins A, Carroll J, West C, Barraclough R, Rudland P: Association of SIOOA4 and osteopontin with specific prognostic factors and survival of patients with minimally invasive breast cancer. Clin Cancer Res 2006, I 2: I 192-1200.

46. Alter $O$, Brown PO, Botstein D: Generalized singular value decomposition for comparative analysis of genome-scale expression data sets of two different organisms. Proc Natl Acad Sci USA 2003, 100:335I-3356.

47. Tibshirani R, Hastie T, Narasimhan B, Chu G: Diagnosis of multiple cancer types by shrunken centroids of gene expression. Proc Natl Acad Sci USA 2002, 99:6567-6572.

48. Hubbard T, Barker D, Birney E, Cameron G, Chen Y, Clark L, Cox T, Cuff J, Curwen V, Down T, et al.: The Ensembl genome database project. Nucleic Acids Res 2002, 30:38-4I.

49. Balanda KP, MacGillivray HL: Kurtosis: a critical review. Am Stat 1988, 42: III-II9.

50. Snedecor GW, Cochran WG: Statistical Methods 6th edition. Ames, IA: lowa State University Press; 1967.

5I. Schwarz G: Estimating the dimension of a model. Annls Stat 1978, 6:46|-464.

52. Yeung KY, Fraley C, Murua A, Raftery AE, Ruzzo WL: Model-based clustering and data transformations for gene expression data. Bioinformatics 200I, I7:977-987.

53. Attias $\mathrm{H}$ : Inferring parameters and structure of latent variable models by variational bayes. In Proceedings of the 15th Conference on Uncertainty in Artificial Intelligence; 30-31 July 1999; Stockholm, Sweden San Francisco, CA: Morgan Kaufmann; 1999:21-30.

54. MacKay DJ: Developments in probabilistic modelling with neural networks-ensemble learning. In Neural Networks: Artificial Intelligence and Industrial Applications. Proceedings of the 3rd Annual Symposium on Neural Networks: 14-15 September 1995; Nijmengen, The Netherlands Berlin: Springer; 1995:191-198.

55. R Development Core Team: $R$ : a language and environment for statistical computing 2003 [http://www.R-project.org]. Vienna, Austria: R Foundation for Statistical Computing 